Perinatology pISSN 2508-4887 • elSSN 2508-4895
Case report

Perinatology Vol. 30, No. 1, March, 2019 https://doi.org/10.14734/PN.2019.30.1.27

\begin{abstract}
Hye Sung Hwang, MD,
Ja Hyun Koo, MD,

Shin Young Kim, MD,

Ra Yon Kim, MD, PhD,

Jeong Ha Wie, MD, PhD,

Hyun Sun Ko, MD, PhD,

In Yang Park, MD, PhD,

Jong Chul Shin, MD, PhD
\end{abstract}

Department of Obstetrics and Gynecology, College of Medicine, The Catholic University of Korea, Seoul, Korea
Received: 28 May 2018

Revised: 6 September 2018

Accepted: 8 October 2018

Correspondence to

Shin Young Kim, MD

Department of Obstetrics and

Gynecology, College of Medicine,

The Catholic University of Korea,

222 Banpo-daero, Seocho-gu, Seoul

06591, Korea

Tel: +82-2-2258-3021

Fax: +82-2-595-1549

E-mail: genius.sinying@gmail.com

Copyright@ 2019 by The Korean Society of Perinatology

This is an Open Access article distributed under the terms of the Creative Commons Attribution Non-Commercial License (http://creativecommons.org/ license/by-nc/4.0/), which permits unrestricted non-commercial use, distribution, and reproduction in any medium, provided that the original work is properly cited.

\section{The Clinical Importance of the Prenatal Diagnosis of Fetal Scalp Hemangioma}

Scalp hemangioma is a rare disease of the extracranial mass in the fetus. Here, we demonstrate a case of fetal scalp hemangioma as evaluated by prenatal ultrasonography and magnetic resonance image (MRI). In this case, a fetal scalp mass measuring $6.3 \times 3.7 \mathrm{~cm}$ was found at 32 weeks of gestation by ultrasonography. The possibility of fetal hemangioma was considered through 4D power Doppler ultrasonography and MRI. At 36+6 weeks of gestation, cesarean section was performed in order to avoid possible traumatic injury to the fetal scalp mass. Postnatally, the baby was diagnosed with scalp hemangioma, which was then treated with beta blockers. It is important to diagnose hemangioma in the prenatal stage, because such an early diagnosis can help plan the method and timing of delivery so as to reduce any risk of traumatic damage to the tumor. In addition, it can aid the prognosis and treatment of the neonate.

Key Words: Fetus, Magnetic resonance imaging, Ultrasonography, doppler, Prenatal diagnosis, Hemangioma

\section{Introduction}

Congenital scalp hemangioma is a very rare disease. The sonographic appearance and Doppler characteristics of hemangioma are variable and sometimes difficult to differentiate from fetal teratoma or encephalocele. ${ }^{1,2}$ We report a case of fetal scalp hemangioma found by prenatal ultrasonography and evaluated by magnetic resonance image (MRI) and 4D power Doppler ultrasonography. It is important to suspect and diagnose fetal scalp hemangioma as early as possible, because such an early diagnosis can help decide the delivery mode and timing of delivery so as to reduce the risk of fetal injury or bleeding caused by labor or vaginal delivery.

\section{Case}

A 43-year-old multigravida woman was referred to Seoul St. Mary's Hospital at 32+4 weeks of gestation with a fetal scalp mass. The patient's antenatal examination was unremarkable until the scalp mass was found by third-trimester screening ultrasonography performed at a local clinic. An integrated test performed at the second-trimester showed low risks for the detection of Down syndrome, Edward syndrome, and neural tube defect. No other anomalies were observed on level II ultrasonography performed at 25+4 weeks of gestation. The ultrasonography at our hospital, performed with a Voluson E10 (GE healthcare, Tiefenbach, Austria), showed a single female fetus in cephalic presentation with fetal biometry appropriate for the gestational age. Ultrasonography showed a $6.3 \times 3.7$ $\mathrm{cm}$ homogeneously echogenic solid mass covering the fronto-parieto-occipital area of the fetal scalp (Fig. 1A, 1C). Color/power Doppler images identified prominent vascular flow 
at the periphery of the tumor (Fig. 1B). Neither skull defects nor intracranial extension were visible on ultrasonography. For further evaluation, fetal MRI was performed using the Ingenia 3.0 Tesla MR imaging system (Philips Healthcare, Eindhoven, the Netherlands) to obtain T2-weighted images (TR 1614.2 $\mathrm{ms} / \mathrm{TE} 80.0 \mathrm{~ms}$ ), and these showed a $3.0 \times 6.0 \times 5.0 \mathrm{~cm}$ mass with mixed low and high signal intensities and internal signal void, suggesting hemorrhage or calcification in the tumor (Fig. 1D). The mass had a broad base on the scalp extending to the left periorbital region. No skull defects or abnormal intracranial lesions were noted. Considering the mixed signal intensity in the MRI and prominent flow signals in the ultrasonography Doppler study, prenatal diagnosis considered fetal scalp hemangioma.

Until 36 weeks of gestation, follow-up ultrasonography examinations showed no significant change in mass size. An early delivery was planned for $36+6$ weeks of gestation, because spontaneous labor may cause massive compression that could result in traumatic bleeding or damage to the fetal scalp mass; thus cesarean delivery was performed in order to avoid trau- matic hemorrhage of the mass and the risk of dystocia. A live baby girl weighing 2,970 g was delivered with Apgar scores of 6 and 8 at one and five minutes, respectively. A $9.0 \times 7.0 \mathrm{~cm}$ well-defined mass was present in the left fronto-parietal area (Fig. 2A, 2B).

Postnatal MRI with Achieva 1.5 Tesla MR imaging system (Philips Healthcare) (TR $5235.9 \mathrm{~ms} / \mathrm{TE} 100.0 \mathrm{~ms}$ ) showed that the fetal scalp mass had a homogenous appearance and intense enhancement. The lesion was located between the orbicularis occuli and the temporalis muscle and extended to the soft tissue of the left lateral orbit. Compressive contour deformity and mild erosive thinning of adjacent skull were observed as well. The tumor showed intermediate signal intensity on T1weighted images (Fig. 2D) and high signal intensity on T2weighted images (Fig. 2C). MRI also demonstrated a signal void, suggesting the tumor's vascular structure. There was multifocal low signal intensity on the susceptibility weighted imaging, suggesting hemangioma with petechial hemorrhage or thrombus (Fig. 2E). Therefore, the postnatal diagnosis of the fetal scalp mass was hemangioma. After consulting with
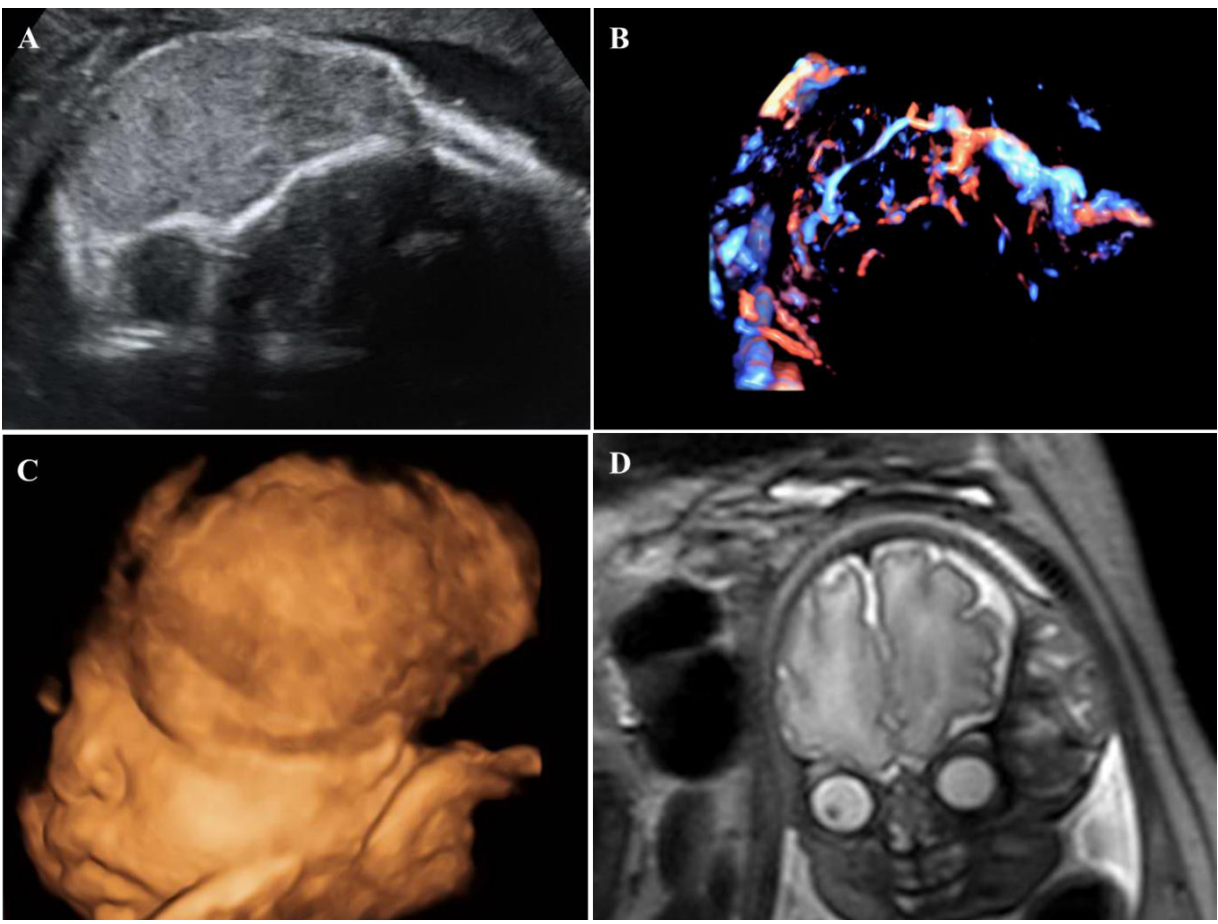

Fig. 1. Antenatal ultrasonography and MRI of the fetal scalp mass at 32 weeks of gestation. (A) Axial view of the fetal scalp mass with gray-scale ultrasonography. (B) 4D power Doppler image of the fetal scalp mass. (C) 3D ultrasonography of the fetal face. (D) T2-weighted image of fetal MRI, coronal view. MRI, magnetic resonance image. 

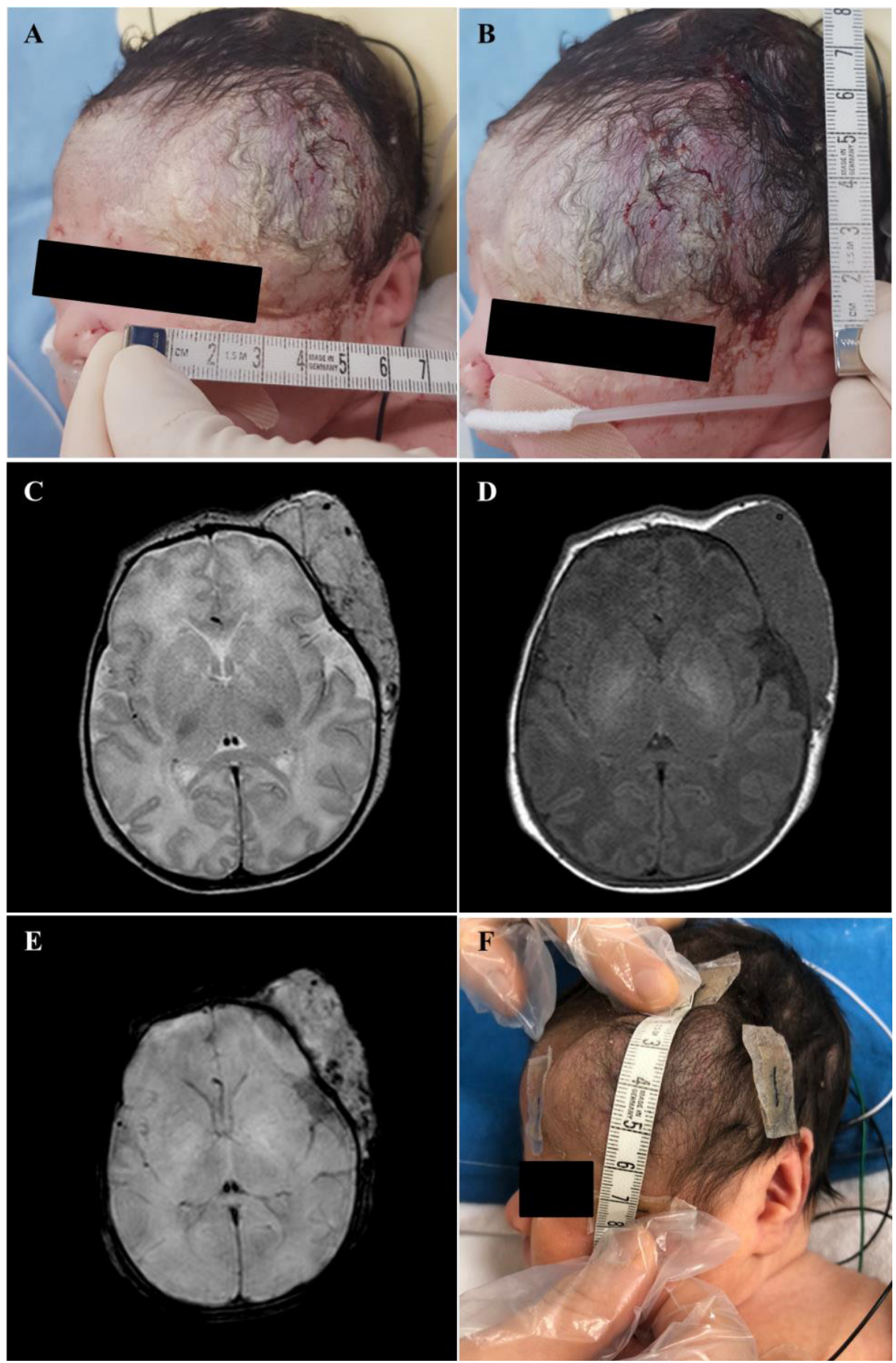

Fig. 2. Postnatal MRI and photographs showing the scalp hemangioma. (A, B) Photographs of the scalp hemangioma at the time of delivery. (C) Postnatal T2-weighted axial MRI. (D) Postnatal T1weighted axial MRI. (E) Postnatal susceptibility-weighted MRI. (F) Photograph of the scalp hemangioma after one week of treatment. MRI, magnetic resonance image.

a neurosurgery team and general pediatric surgery team, the baby was treated with beta blockers without excision in order to decrease the size of the mass. Following seven days of treat- ment, the mass size had decreased from $9.0 \times 7.0 \mathrm{~cm}$ to $7.3 \times 6.8$ $\mathrm{cm}$ (Fig. 2F). The dose of atenolol was gradually increased, and the neonate is currently taking 9 mgs of atenolol twice a day. 
Computed tomography (CT) will be performed after four to five months of medical treatment, and surgical excision may be performed based on those $\mathrm{CT}$ results.

\section{Discussion}

Congenital hemangiomas are rare benign vascular tumors that are fully developed at birth..$^{3-5}$ They have two major subtypes: rapidly involuting congenital hemangiomas (RICHs) and non-involuting congenital hemangiomas (NICHs), based on their clinical prognosis after birth. ${ }^{3-5}$ RICHs are more common and usually involute spontaneously within the first 12-14 months of life. ${ }^{5,6}$ In contrast, NICHs typically require surgical excision..$^{3-5}$ Clinically, RICHs and NICHs have overlapping characteristics and are difficult to differentiate at the prenatal stage. ${ }^{7}$ The most common locations of congenital hemangioma are the neck, head, and limbs; ${ }^{3}$ scalp hemangiomas are relatively rare.

The ultrasonography features of congenital hemangioma vary depending on the sizes and types of vessels present, the amount of arteriovenous shunting, and the degree of endothelial proliferation. ${ }^{8}$ Sonographic characteristics range from solid masses with homogeneous echogenic patterns to mixed solidcystic lesions with heterogeneous appearance. ${ }^{5,9,10}$ Frequently, fetal hemangiomas demonstrate calcification on ultrasonography.,11 Doppler ultrasonography depicts vascular blood flow or microshunts within the tumor. ${ }^{3,11}$

Recently, fetal MRI has been used more frequently as an adjunctive diagnostic tool for the differential diagnosis of fetal scalp masses. On MRI, congenital hemangiomas present as well-defined masses with mixed signal intensities and a reticulated appearance on T1- and T2-weighted images. ${ }^{3}$

Once a fetal scalp mass has been found prenatally, several diseases should be considered in differential diagnosis, including teratoma, hemangioma, encephalocele, cystic hygroma, lymphangioma, and mesenchymal sarcoma. ${ }^{3,11}$ On ultrasonography, teratomas appear as masses with calcifications and solid or cystic components. ${ }^{12}$ Encephalocele is the most common posterior midline extracranial mass, and shows skull defects and associated cerebral abnormalities such as hydrocephalus. ${ }^{1}$ Lymphangiomas and cystic hygromas appear on prenatal ultrasonography as fetal neck masses of various sizes with cystic components and multiple septa. ${ }^{3}$ The differential diagnosis of those tumors is important because they differ in prognosis and treatment. In the case of congenital hemangioma, spontaneous labor or vaginal delivery may cause traumatic hemorrhage in a tumor, so an elective cesarean section should be considered prior to labor.

In our case, a fetal scalp hemangioma on the left frontoparieto-occipital area was found at 32 weeks of gestation, and there were no associated anomalies or other complications such as fetal cardiac dysfunction. On ultrasonography, the fetal scalp mass was a homogeneously echogenic solid mass with prominent vascular flow. Prenatal MRI indicated that the extracranial mass was heterogeneous in appearance with an internal signal void that suggested hemorrhage or calcification in the tumor. Early cesarean section was performed at $36+6$ weeks of gestation in order to avoid traumatic hemorrhage of the mass as well as the risk of dystocia. Postnatal MRI showed intermediate signal intensity on T1-weighted images and high signal intensity on T2-weighted images, which are characteristic features of infantile hemangioma. ${ }^{2,10}$ Following consultation, the baby was treated with beta blockers. The exact mechanism of the benefits of using beta blockers for congenital hemangioma is yet to be elucidated, although it has been suggested that vasoconstriction, the inhibition of angiogenesis, and the induction of apoptosis of capillary endothelial cells inhibit tumor growth. ${ }^{13}$ As has been reported in previous studies, fetal scalp hemangioma may have spontaneous resolution after birth. ${ }^{3,6}$ However, in this case, considering the size and position, it could affect the visual axis when the size has increased. Moreover, there was a risk of complications such as bleeding. Therefore, after consultation with the parents, the neonate was treated with medication.

In conclusion, the proper diagnosis of hemangioma with antenatal ultrasonography and MRI is crucial once a scalp mass has been found in utero. This can help to decide the timing and method of delivery and thus reduce the risk of traumatic hemorrhage during vaginal delivery.

\section{Conflict of interest}

No potential conflict of interest relevant to this article was reported. 


\section{References}

1) Bronshtein M, Bar-Hava I, Blumenfeld Z. Early second-trimester sonographic appearance of occipital haemangioma simulating encephalocele. Prenat Diagn 1992;12:695-8.

2) Yoshida S, Kikuchi A, Naito S, Nakamura H, Hayashi A, Noguchi M, et al. Giant hemangioma of the fetal neck, mimicking a teratoma. J Obstet Gynaecol Res 2006;32:47-54.

3) Jo EJ, Choi SJ, Oh SY, Park BK, Roh CR, Kim JH. Complete involution of prenatally-diagnosed fetal scalp hemangioma. Taiwan J Obstet Gynecol 2014;53:86-9.

4) Boull C, Maguiness SM. Congenital hemangiomas. Semin Cutan Med Surg 2016;35:124-7.

5) Elia D, Garel C, Enjolras O, Vermouneix L, Soupre V, Oury JF, et al. Prenatal imaging findings in rapidly involuting congenital hemangioma of the skull. Ultrasound Obstet Gynecol 2008;31:572-5.

6) Ozcan UA. Rapidly involuting congenital hemangioma: a case of complete prenatal involution. J Clin Ultrasound 2010;38:85-8.
7) Amouri $M$, Mesrati $H$, Chaaben $H$, Masmoudi $A$, Mseddi $M$, Turki $H$. Congenital hemangioma. Cutis 2017;99:E31-3.

8) Bulas DI, Johnson D, Allen JF, Kapur S. Fetal hemangioma. Sonographic and color flow Doppler findings. J Ultrasound Med 1992;11:499-501.

9) Miyakoshi K, Tanaka M, Matsumoto T, Hattori Y, Minegishi K, Ishimoto H, et al. Occipital scalp hemangioma: prenatal sonographic and magnetic resonance images. J Obstet Gynaecol Res 2008;34(4 Pt 2):666-9.

10) Kramer LA, Crino JP, Slopis J, Hankins L, Yeakley J. Capillary hemangioma of the neck: prenatal MR findings. AJNR Am J Neuroradiol 1997;18:14324.

11) Viora E, Grassi Pirrone P, Comoglio F, Bastonero S, Campogrande $M$. Ultrasonographic detection of fetal cranio-facial hemangioma: case report and review of the literature. Ultrasound Obstet Gynecol 2000;15: 431-4.

12) Kadlub N, Touma J, Leboulanger N, Garel C, Soupre V, L'Herminé AC, et al. Head and neck teratoma: from diagnosis to treatment. J Craniomaxillofac Surg 2014;42:1598-603.

13) Shah S, Frieden IJ. Treatment of infantile hemangiomas with betablockers: a review. Skin Therapy Lett 2013;18:5-7. 